\title{
Civilisations
}

Revue internationale d'anthropologie et de sciences

humaines

$41 \mid 1993$

Mélanges Pierre Salmon II

\section{Histoire structurale d'une religion africaine}

Le culte des Cwezi et des Imandwa dans la région des Grands Lacs

\section{Luc de Heusch}

\section{(2) OpenEdition}

\section{Journals}

Édition électronique

URL : http://journals.openedition.org/civilisations/1609

DOI : 10.4000/civilisations. 1609

ISSN : 2032-0442

Éditeur

Institut de sociologie de l'Université Libre de Bruxelles

\section{Édition imprimée}

Date de publication : 1 septembre 1993

Pagination : $19-50$

ISBN : 2-87263-094-5

ISSN : 0009-8140

\section{Référence électronique}

Luc de Heusch, "Histoire structurale d'une religion africaine », Civilisations [En ligne], 41 | 1993, mis en ligne le 30 juin 2009, consulté le 19 avril 2019. URL : http://journals.openedition.org/civilisations/1609 ; DOI : 10.4000/civilisations. 1609

Ce document a été généré automatiquement le 19 avril 2019

(c) Tous droits réservés 


\title{
Histoire structurale d'une religion africaine
}

Le culte des Cwezi et des Imandwa dans la région des Grands Lacs

\author{
Luc de Heusch
}

1 J'avais entrepris en 1966, dans un livre consacré au Rwanda et à la civilisation interlacustre, de montrer que l'analyse structurale, loin d'être incompatible avec l'interprétation historique, pouvait au contraire lui être d'un grand secours. J'avais repris cette hypothèse dans Rois nés d'un coeur de vache (1982). Cette position me valut une levée de boucliers de la part des ethno-historiens ${ }^{1}$. C'est plus spécialement à mon interprétation du culte de possession connu au Rwanda sous le nom de kubandwa et répandu sous des formes variables dans toute l'aire culturelle des Grands-Lacs (au Burundi, en Ouganda comme en Tanzanie) que Iris Berger s'en prend tout au long de son livre Religion and Resistance, East African Kingdoms in the Precolonial Period non sans reprendre subrepticement certaines de mes idées (Berger, 1981). L'enjeu théorique est tel que je n'hésiterai pas, dans ma tardive réponse, à entrer dans un certain nombre de précisions techniques qui risquent d'irriter le lecteur non averti. 


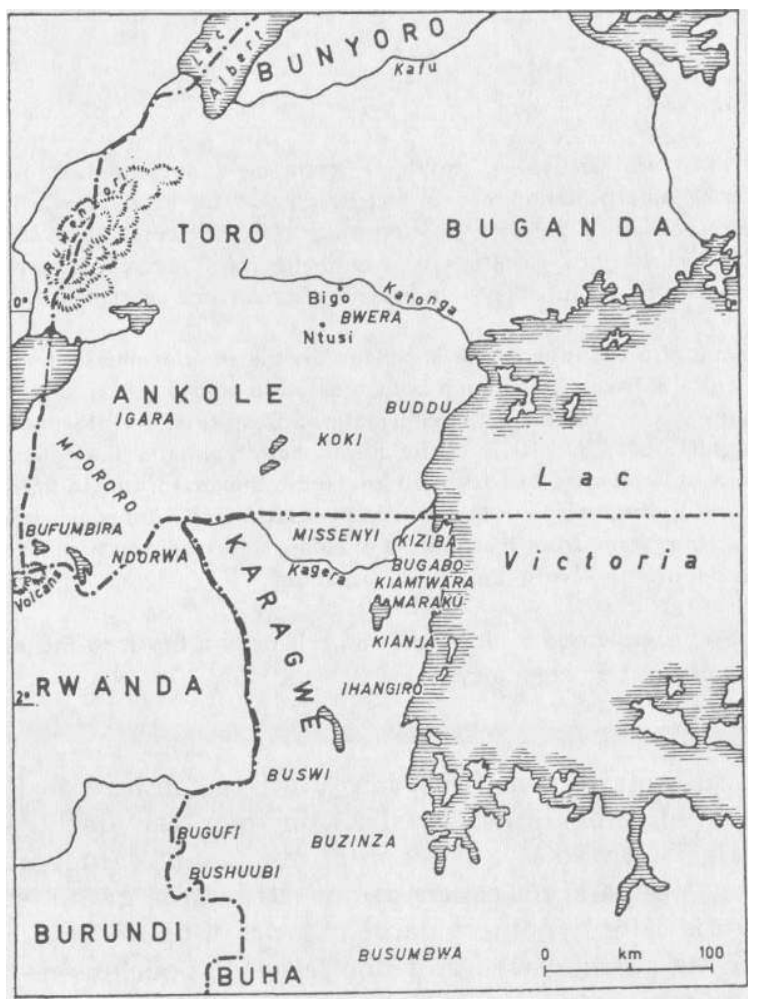

D'un point de vue général, Berger me reproche d'avoir institué en un ensemble arbitraire les divinités et les mythes locaux associés à ce culte et d'avoir montré qu'ils étaient liés entre eux par des transformations structurales, dont j'ai tenté d'esquisser la diachronie. Berger dénonce « l'erreur qui consiste à croire que les esprits, le mythe et le rite forment un système unifié qui se répand à travers le temps et l'espace» (Berger, 1981, p. 66). De quoi s'agit-il?

\section{La geste des Cwezi (Berger, 1981, pp. 127-134)}

Nous sommes sur les hauts plateaux de l'Afrique centrale et orientale, qui s'étendent entre les lac Mobutu (ex-Albert) au nord, Edouard et Kivu à l'ouest, Victoria à l'est, Tanganyika au sud. Dans cette région favorable à l'élevage du gros bétail, des pasteurs et des agriculteurs se sont rencontrés au sein de royaumes d'importance variable, qui n'ont pas résisté aux tourmentes de la décolonisation. Au Nyoro et au Nkole (Ouganda), une tradition orale remarquablement constante fait état d'un âge d'or - celui du royaume de Kitara - associé au peuple cwezi ${ }^{2}$ disparu dans des circonstances mystérieuses, en s'enfonçant sous terre ou dans un lac. Ces personnages légendaires communiquent cependant toujours avec des initiés, hommes ou femmes, qui sont leurs médiums (mbandwa). Par ailleurs, les rois historiques du Nyoro comme ceux du Nkole prétendent se rattacher au dernier souverain cwezi, Wamara, par une voie indirecte, bien qu'ils soient manifestement des nouveaux-venus. Mais les Cwezi eux-mêmes présentent leurs deux souverains successifs, Ndahura et Wamara, comme les successeurs (par l'intermédiaire d'une femme) d'une dynastie précédente, celle des Tembuzi. Ce qu'il y a de remarquable, c'est que les Cwezi comme les Tembuzi sont des créatures associées au monde chthonien. Nyamiyonga, le maître du royaume inférieur, désira conclure un pacte du sang avec Isaza, le premier roi tembuzi de Kitara. Hésitant, Isaza se fit remplacer par son serviteur Bukuku. Furieux, Nyamiyonga dépêcha sa fille auprès de Isaza avec mission de le séduire. 
Enceinte de six mois, la belle retourna dans le monde souterrain où, trois mois plus tard, elle mit au monde un fils, Isimbwa. Pour attirer auprès de lui le père de l'enfant, Nyamiyonga lui envoya un couple de bovins, qui entraîna bientôt Bihogo, la vache préférée de Isaza, dans le monde inférieur. Isaza suivit sa vache et c'est ainsi qu'il retrouva sa femme et son fils auprès desquels il demeura. Devenu adulte, Isimbwa décida de découvrir le royaume terrestre que son père avait confié à la garde de Bukuku. Des devins avaient annoncé à celui-ci que sa fille lui porterait malheur. C'est pourquoi il la tenait enfermée dans sa maison après lui avoir fait couper un sein et arracher un oeil.

Isimbwa parvint jusqu'à elle déguisé en chasseur et la séduisit. Elle mit au monde un fils, Ndahura. Bukuku fit jeter le nouveau-né dans la rivière, mais un potier le sauva et l'éleva. Plus tard, Ndahura tua son grand-père maternel et reprit possession du trône que celui-ci avait usurpé. Ndahura fit de nombreuses conquêtes militaires. Son père Isimbwa vint le rejoindre et confirma sa légitimité; Ndahura lui confia le gouvernement de deux provinces. L'empire de Kitara s'étendit sur une large partie de l'Ouganda occidental englobant notamment le Nkore. Ndahura disparut au cours d'une campagne en Tanzanie, il fut mystérieusement avalé par la terre. Wamara, le fils aîné de Ndahura, qui avait partagé le pouvoir avec son père, prit la succession. Il mit fin à diverses rébellions, mais son règne fut troublé. Une série de présages funestes annoncèrent la fin des Cwezi. Ils décidèrent alors de quitter le pays. La tradition orale du Nkore qui reprend, avec de légères variantes, le même récit précise que les $\mathrm{C}$ wezi s'enfoncèrent sous terre ou dans les profondeurs d'un lac.

5 Un premier mot de commentaire. L'ensemble de ces récits obéit à une armature symbolique évidente: associer au monde souterrain, par les femmes, une première dynastie -, celle des Tembuzi, représentée par deux rois (Isaza et Isimbwa) - prélude à une dynastie terrestre (celle des Cwezi) qui comprend aussi deux souverains (Ndahura et Wamara). Ceux-ci sont appelés à revenir en leur lieu d'origine : le monde inférieur. C'est bien une histoire en miroir que raconte la geste des Tembuzi-Cwezi. Au début des temps, le premier roi tembuzi suit sa vache préférée Bihogo jusque dans le monde souterrain. C'est un animal portant le même nom qui est à l'origine de la disparition sous terre des Cwezi à l'époque du dernier souverain Wamara. Voici l'histoire. Mugenyi, le neveu de Wamara avait fait serment de ne pas survivre à sa vache préférée, Bihogo. Or celle-ci mourut et Wamara décida de consulter les auspices. Ceux-ci découvrirent que Bihogo n'avait pas d'entrailles. Ils n'osèrent révéler cette terrible nouvelle à Wamara. Un étranger s'en chargea, prophétisant que le règne des Cwezi était terminé. Mugenyi semblait avoir oublié son serment de mourir avec la vache. Ce parjure eut le don d'irriter sa propre tante. Humilié par les propos désagréables qu'elle lui tint, Mugenyi prit la décision de quitter ce pays maudit où les femmes osaient se moquer des hommes. Wamara approuva la décision de son neveu et tous les Cwezi se rallièrent au projet de départ. Ils rassemblèrent leurs femmes, leurs troupeaux et leurs biens. Ils s'en allèrent et on ne les revit plus jamais au Nkore (Mungonya, 1958).

6 Selon une autre version, les Cwezi seraient partis vers le Karagwe, une région située en pays haya, au sud du Nkore (Tanzanie), où ils disparurent dans un lac (Oberg, 1970, p. 124). Or la tradition haya reprend, pour expliquer la disparition de Wamara, la thématique même du départ dans le monde souterrain de Isaza, le roi tembuzi. En voici le résumé d'après Césard qui dit avoir vérifié l'authenticité de l'histoire auprès de plusieurs informateurs (Césard, 1927, pp. 447-455). 
7 Deux chefs, Irungu (devenu esprit de la brousse) et Mugasha (devenu esprit du lac) vivaient avec Wamara. Un chacal glapit dans la cour à minuit. Wamara décida de le poursuivre avec ses deux compagnons. Le chacal entraîna les chasseurs en forêt, puis dans un souterrain conduisant au royaume infernal du roi Kintu (appelé Kintu d'Igaba). Kintu offrit des cadeaux à Wamara: de la bière de banane, du lait, des vaches et des chèvres. Les terriens restèrent neuf jours auprès de Kintu. Mugasha fit une provision de semences de légumes et ils retournèrent sur terre nantis de ces présents. Kintu cependant avait recommandé à Wamara de ne pas oublier son hôte généreux. En voyant sortir des vaches du souterrain, les hommes de Wamara furent effrayés, puis émerveillés. Wamara confia le bétail à la garde de son frère aîné Ryangombe. Des années passèrent et Wamara ne remercia pas Kintu. Au contraire, il fit boucher l'entrée du souterrain. Alors Kintu envoya Rufu, la mort, qui réclama les vaches. Wamara, loin de s'exécuter, fit rouer de coups le messager de Kintu. Rufu s'enfuit mais réussit plus tard à attirer une vache blanche, particulièrement précieuse, dans une fosse marécageuse. Or, jadis, Wamara avait fait le serment de mourir avec elle. Il se jeta dans le gouffre avec Irungu et tous ses compagnons. Ils ne réapparurent plus jamais, mais jusqu'à nos jours les fidèles initiés aux mystères religieux de Wamara sont périodiquement visités par les esprits des Cwezi disparus.

Césard précise que le gouffre appelé Imara, où ces événements extraordinaires sont censés s'être déroulés, se situerait « sur les confins du Karagwe ».

Le thème de la chasse mérite une attention particulière. Dans un chant rituel nyoro, Wamara est célébré comme chasseur: "C'est lui, vraiment, qui chasse les animaux sauvages des champs. C'est lui, Wamara... » (Berger, 1981, p. 19). Le récit nkore attribue la même qualité à l'ensemble des Cwezi, qui se voient dotés de pouvoirs magiques considérables (Oberg, 1970, p. 123 ; édition originale 1940). C'est aussi comme chasseur que Isimbwa, le deuxième roi tembuzi, né dans le monde inférieur, surgit sur terre. Inverssément, c'est comme chasseur que Wamara, deuxième roi cwezi, disparaît sous terre dans la geste haya que l'on vient de lire. Dans cet épisode, qu'il n'y a aucune raison de séparer de l'ensemble de la geste cwezi dont il n'est qu'une variante, la figure centrale du roi Wamara nous est présentée à la fois comme éleveur (il reçoit le bétail du monde inférieur) et comme chasseur. Il cumule ainsi deux caractéristiques précédemment disjointes : il est un amateur de bétail piégé par le maitre du monde inférieur comme son arrière-grand-père Isaza, et un chasseur comme son grand-père Isimbwa. En revanche tous s'accordent à reconnaître en Ndahura un grand guerrier (Berger, 1981, p. 20). La structure narrative de cet ensemble mythique fait se succéder les fonctions symboliques suivantes :

\begin{tabular}{|l|l|}
\hline Isaza : & pasteur \\
\hline \hline Isimbwa : & chasseur \\
\hline Ndahura : & guerrier \\
\hline Wamara : & pasteur et chasseur \\
\hline
\end{tabular}

Si Wamara est célébré dans la geste cwezi comme "celui qui complète " l'oeuvre de Ndahura le conquérant (Wrigley, 1973, p. 227), son règne se caractérise cependant par un 
affaiblissement de la puissance des Cwezi que Ndahura avait imposée par les armes. Les fonctions pastorale et cynégétique qu'il assume dans le mythe sont marquées négativement : la vache Bihogo est porteuse d'un présage funeste et la dernière chasse de Wamara l'entraîne dans le royaume des morts. On comprend qu'en pays haya, ce génie tutélaire du bétail soit aussi le maître de l'au-delà (Césard, 1937, pp. 17-18).

11 J'ai souligné ailleurs la valeur de l'opposition chasseur/guerrier dans les gestes de fondation de l'Etat en Afrique centrale (L. de Heusch, 1972 et 1982). J'attirais notamment l'attention sur le fait que le héros chasseur est souvent dans le mythe l'annonciateur de la royauté sacrée que fonde ultérieurement un héros guerrier. C'est le cas chez les LubaLunda comme dans la région interlacustre qui nous concerne ici, au Rwanda.

C'est encore un chasseur étranger, un nilotique, qui viendra fonder au Nyoro la nouvelle dynastie, celle des Bito, après la disparition des Cwezi. Wrigley a bien montré que l'irruption de Rukidi, le premier roi bito, s'intègre à l'ensemble mythique comprenant les Tembuzi et les Cwezi (Wrigley, 1973). Il observe à juste titre que le nom de Rukidi renvoie au terme kidi, qui désigne les hommes de la brousse; Rukidi le sauvage, le chasseur aux longs cheveux, s'oppose donc aux pasteurs cwezi hypercivilisés que la tradition crédite de l'introduction de la royauté sacrée. C'est une femme du clan cwezi, qui n'a pas suivi les siens dans l'exil, qui le civilise; elle lui apprend à boire le lait. Wrigley se borne à interpréter cet épisode mythique comme un rite d'initiation royal. Il n'aperçoit pas que cet apprentissage fait en quelque sorte passer Rukidi de l'espace de la nature (marqué par la chasse) à l'espace culturel. Ce mythème peut être interprété comme un retour à la civilisation après l'effondrement culturel que constitue la disparition des Cwezi engloutis par les éléments naturels. L'arrivée de Rukidi l'étranger, c'est aussi la fin de deux dynasties marquées par l'autochtonie.

Rukidi est présenté comme un jumeau. Ce trait, dont Wrigley avoue ne pas comprendre les liens avec la royauté sacrée, ne fait qu'accentuer la qualité d' « homme de la brousse » de Rukidi. En effet, la gémelléité, toujours sacralisée positivement ou négativement, apparait souvent en Afrique comme l'irruption dans l'ordre culturel humain d'une fécondité naturelle, excessive, animale ${ }^{3}$. Rukidi, c'est le retour du sauvage, la régénération de la royauté cwezi déliquescente par l'irruption des forces naturelles fécondantes. Car la puissance magique chthonienne des rois cwezi s'est épuisée.

Ces fils jumeaux seraient nés de l'union d'un prince cwezi, le propre frère de Wamara, et d'une femme nilotique (W.K., 1935). Le mythe de Rukidi s'inscrit donc bien dans la continuité de la geste cwezi qu'il vient parachever. Il ne saurait être réduit, comme le pense Wrigley, au passage de l'âge des dieux à l'âge des hommes. Au contraire, l'insertion de Rukidi dans la généalogie des Cwezi souligne bien la continuité de la royauté sacrée dans la discontinuité.

Après le départ des Cwezi du Nkore, c'est aussi un nouveau venu, Ruhinda, le chef d'un clan hinda apparenté aux Bito nilotiques, qui prend le pouvoir dans ce royaume (L. de Heusch, 1966, pp. 29-30). Cette fois le fondateur de la nouvelle dynastie est présenté comme le fils bâtard de Wamara (Oliver, 1959). L'histoire s'articule donc avec le mythe, qui en constitue en quelque sorte la charte de légitimation.

Prenons un peu de distance et comparons cet ensemble mythique à celui du Rwanda. Dans Rois nés d'un coeur de vache, j'ai cru pouvoir montrer que la pseudo histoire dynastique constitue jusqu'à une époque fort récente (XVII ${ }^{\mathrm{e}}$ siècle) un ensemble de mythes dont la cohérence ne se laisse déchiffrer que si l'on réduit leur diachronie à une synchronie 
structurale comme je viens de le faire pour la geste cwezi (L. de Heusch, 1982). La dynastie rwandaise est d'origine céleste. Ce n'est qu'au prix d'une longue et constante politique matrimoniale que ces rois sans royaume, «tombés du ciel », s'enracinent, acquièrent une autochtonie qui leur faisait défaut. L'un de ces êtres, Gihanga le Fondateur, renforce cette autochtonie en épousant une princesse renge. Les Renge sont censés avoir été la maison règnante du clan autochtone singa, et son hégémonie s'est étendue longtemps sur la moitié occidentale du pays. Or il se fait que les Renge, comme les Tembuzi-Cwezi d'Ouganda, sont étroitement associés au monde souterrain. Ils disparaissent, noyés dans les profondeurs de la terre (L. de Heusch, 1982, p. 86). Le fait curieux est que Gihanga, le fondateur mythique de la dynastie historique du Rwanda, s'unit clandestinement à une princesse renge dont le père surveillait étroitement sa conduite. Ceci n'est pas sans rappeler la conduite de Bukuku dans la geste tembuzi-cwezi : cet usurpateur qui règne sur terre en lieu et place d'Isaza, disparu dans le monde inférieur, tient sa fille rigoureusement enfermée. Dans les deux cas, c'est un chasseur (Gihanga ou Isimbwa) qui la séduit, garantissant ainsi la continuité dynastique. Mais dans le mythe rwandais, Gihanga, descendant lointain du roi du Ciel (Nkuba la Foudre), est à la recherche d'une légitimité royale chthonienne qui lui fait défaut, tandis que dans la chronique nyoronkole, c'est un prince né dans le monde souterrain qui fait surface pour réclamer le trône de son père. C'est par le même stratagème que la fille de Bukuku et le chasseur Isimbwa donnent naissance au grand Ndahura, le fondateur du Kitara et que la fille du roi renge engrossée par le chasseur Gihanga enfante l'ancêtre éponyme de la dynastie rwandaise, Kanyarwanda, qui se trouve être aussi à l'origine de quelques royaumes voisins (L. de Heusch, 1982, pp. 36-37).

17 C'est donc par l'intermédiaire d'une femme que la dynastie céleste du Rwanda se rattache à la dynastie autochtone des Renge. C'est aussi par une femme - sa grand-mère paternelle - que Ndahura le premier roi cwezi se rattache aux puissances chthoniennes. Mais l'affirmation de l'autochtonie est beaucoup plus marquée dans le cas des Cwezi, qui viennent $\mathrm{du}$ monde souterrain et y retournent alors que la tradition royale rwandaise raconte le triomphe d'une dynastie d'origine céleste. Les gestes des royaumes septentrionaux (Nyoro, Nkore) ne racontent évidemment pas la même histoire que la geste royale rwandaise. Mais elles se développent d'évidence sur un fond mythique commun. Il est remarquable à cet égard que dans les deux traditions, la richesse bovine jaillisse du monde souterrain. On comparera l'origine des vaches dans le mythe de Wamara-Kintu (voir plus haut) à un récit rwandais relatif à Gihanga (L. de Heusch, 1982, pp. 52-54).

Il est de plus en plus clair que la mythologie, bien que soumise au flux de l'histoire, tend à constituer sur des espaces plus ou moins vastes, et durant un temps plus ou moins long, des ensembles cohérents, contrairement à ce que prétend Iris Berger. Je rejoindrai donc volontiers, sur ce point précis, la position théorique générale de Wrigley. Mais celui-ci me reproche par ailleurs d'avoir cru, dans mon livre de 1966, à l'historicité de ces mythiques Cwezi. La critique d'Iris Berger rejoint ici la sienne. Il faut donc que je m'explique.

\section{Mythe et Histoire}

Dans le Rwanda et la civilisation interlacustre, je m'efforçais pour la première fois d'appliquer la méthode structurale à un ensemble mythique africain. A vrai dire à cette époque lointaine, j'étais loin d'apercevoir la cohérence de la geste cwezi, telle que je viens 
de l'esquisser. Mon intérêt principal se portait sur un aspect particulier de cette mythologie, la geste rwandaise de Ryangombe. Nkore n'a donc rien qui puisse surprendre. En tout état de cause, il n'est pas interdit non plus de déchiffrer derrière la trame mythique l'écho d'un certain nombre d'événements. C'est un ethnohistorien chevronné, Jan Vansina, qui décrypte, à propos de l'histoire du Rwanda, un "cliché » historique typique de la tradition orale dans cette région: le fils d'un souverain est absent au moment d'un désastre militaire, il vit à l'étranger et revient en triomphateur (Vansina, 1962, p. 51). Cette formule suggère et dissimule en vérité l'avènement d'une nouvelle dynastie soucieuse de légitimité. Or c'est exactement ce schème qui apparaît dans la transmission du pouvoir de Wamara à Ruhinda, l'envahisseur. Selon la tradition du Nkore, ce fils illégitime de Wamara, fondateur présumé de la nouvelle dynastie, était en train de guerroyer au Karagwe lorsque les Cwezi décidèrent d'émigrer... précisément au Karagwe. La contradiction est évidente. Elle travestit sans doute une tout autre réalité : l'écrasement au Karagwe des Cwezi par les Hinda (L. de Heusch, 1966, pp. 31-35). Vansina reprend d'ailleurs la thèse d'oliver : les Cwezi de l'Ouganda furent chassés entre 1450 et 1500 par les Bito nilotiques (Vansina, 1972, p. 105). 
méridionaux du pays haya, parmi lesquels figure le Karagwe, laisse entendre que c'est sur ces confins méridionaux de l'empire du Kitara - où déjà Ndahura disparut mystérieusement - que les Cwezi ont connu leur défaite finale. C'est là en effet que la légende décrit le retour de la dynastie en son lieu d'origine souterrain après l'abandon du Nkore. C'est précisément parce que les nouvelles dynasties (Bito ou Hinda) entendent récupérer l'idéologie royale de leurs prédécesseurs pour fonder sur des bases sacrées anciennes un pouvoir nouveau, usurpé, que toute trace de combat armé a été soigneusement gommée. Il n'en va nullement ainsi lorsque les mêmes rois nilotiques envahissent (sans succès durable) le Rwanda quelques générations après Rukidi: le souvenir des défaites militaires est demeuré cuisant dans la mémoire rwandaise.

En revanche, il y a lieu de croire que le cliché selon lequel le roi Ruganzu Ndori se serait réfugié chez sa tante paternelle au Karagwe au cours d'une période de chaos avant de reconquérir le Rwanda paternel, dissimule l'invasion du pays par un groupe étranger, peut-être hinda, soucieux de s'inscrire dans la continuité dynastique rwandaise (Vansina, 1962, p. 51).

\section{Rectifications chronologiques}

Je n'ai pas l'intention de m'enfermer ici dans la querelle chronologique. Je renoncerai à dater ces événements. Je m'étais basé précédemment sur la chronologie Vansina qui situait le règne de Ruganzu au début du XVII ${ }^{e}$ siècle. Mais cette hypothèse vient d'être bouleversée par une datation au carbone 14 effectuée sur la momie de Cyirima Rujigira, qui fixe la mort de ce sixième successeur de Ruganzu Ndori précisément à cette époque et non, comme le pensait Vansina, au milieu du XVIII ${ }^{e}$ siècle. Van Noten (1972, p. 50) propose 1635 comme date la plus probable pour les ossements. Par ailleurs Berger souligne les difficultés que pose le problème crucial du raccord chronologique de la dynastie bito du Nyoro avec celle du Rwanda (Berger, 1981, pp. 135-136).

Dans l'état actuel de l'information, il est donc très difficile de situer dans le temps la fin de l'empire cwezi et l'arrivée des conquérants nilotiques au Nyoro. J'avais suggéré le cours du XVe siècle en reprenant l'estimation d'Oliver (Oliver, 1955). Il est probable que ces événements se situent plus près de 1400 que de 1500. En tout état de cause, il n'y a pas lieu de renoncer à l'hypothèse, suggérée par les traditions orales du pays haya qui présentent les Hinda comme un sous-clan bito issu des conquérants du Nyoro (L. de Heusch, 1966, pp. 29-30). La conquête ultérieure par les Hinda des petits royaumes haya, sur les marches méridionales de l'empire de Kitara, pourrait donc avoir commencé au cours du XVI ${ }^{e}$ siècle, comme le suggéraient déjà Ford et Hall (1947). Ces remous sur le flan oriental $\mathrm{du}$ bastion montagneux rwandais expliqueraient ainsi le surgissement $\mathrm{du}$ Karagwe de Ruganzu Ndori, le fondateur d'une nouvelle dynastie, à une date que nous nous garderons bien désormais de préciser.

\section{Transformations mythologiques : un problème historique et structural}

28 Au Nyoro, au Nkore comme au Karagwe, les esprits Cwezi reçoivent un culte officiel par l'intermédiaire de médiums attitrés et Wamara s'inscrit dans la généalogie dynastique des nouveaux rois bito ou hinda. Dans les petits royaumes haya de Tanzanie, de nombreux 
clans (de pasteurs ou d'agriculteurs) honorent un esprit cwezi particulier ; mais le nom de Wamara revient le plus souvent. Il est notamment le génie tutélaire du clan royal hinda au Karagwe comme en Ihangiro, au Kiziba et au Kiantwara (Cory \& Hartnoll, 1945, annexe V).

29 Mais un autre nom, celui de Ryangombe, apparaît aussi avec insistance. Neuf clans honorent particulièrement ce Cwezi à qui Wamara aurait confié la garde de son bétail (Césard, 1927, pp. 17-18).

Cependant Ryangombe ne figure pas parmi les parents de Wamara dans la généalogie des Cwezi. Son nom n'est pas repris dans la liste des dix-neuf esprits cwezi établie par Roscoe au Nyoro en 1923 (Roscoe, 1923, pp. 22-28). Au Nkore, il est cité comme chasseur parmi les Cwezi (Oberg, 1970, p. 124). Il appartient à une classe inférieure d'esprits par rapport aux grands Cwezi tels Ndahura et Wamara; ceux-ci sont les maitres, ceux-là leurs serviteurs (Karugire, 1971, p. 84).

31 La figure de Ryangombe, passablement effacée, domine en revanche le mythe et le rituel dans l'aire méridionale de la civilisation interlacustre. Il est le « roi des Imandwa » dans le culte de possession kubandwa au Rwanda, au Burundi et au Buha; il est le fondateur du culte des esprits swezi (transformation phonétique de Cwezi) au Sumbwa et au Nymawezi (L. de Heusch, 1966, pp. 282-294). Dès lors, j'ai été amené à opposer une zone Wamara à une zone Ryangombe. Iris Berger reprend à son compte cette distinction, en oubliant de me créditer de l'avoir établie. Elle se sépare néanmoins radicalement de moi sur le plan de l'interprétation. J'avais montré qu'un processus de transformation structurale permettait de passer de la légende haya (décrivant la disparition sous terre de Wamara et des Cwezi) au mythe rwandais narrant la mort tragique de Ryangombe (tué par un buffle) suivie du suicide de tous ses compagnons (les Imandwa) appelés à séjourner en compagnie des morts au sommet du volcan Karisimbi. Ryangombe comme Wamara sont de grands chasseurs et c'est au cours d'une ultime partie de chasse qu'ils deviennent l'un et l'autre souverains de l'Au-Delà.

C'est bien sur les confins méridionaux de l'aire de domination bito-hinda, c'est-à-dire en pays haya et au-delà, que l'on voit la pensée mythique substituer Ryangombe à Wamara, après avoir éliminé ce dernier de la scène mythique. Plusieurs étapes marquent cette transformation. Dans une version récoltée chez les Haya par Césard, l'on voit d'abord Ryangombe surgir aux côtés de Wamara comme gardien de bétail de celui-ci et esprit protecteur des troupeaux (Césard, 1937, pp. 17-18 et 1927, pp. 447-455).

La seconde étape est un changement spectaculaire de scénario, en pays sumbwa, au sud du Haya, c'est-à-dire au-delà des limites de l'expansion des conquérants hinda. Wamara est un perturbateur à la cour du roi hinda du Karagwe. Ryangombe, un esclave de celui-ci, promet de venir en aide à tous ceux qui l'imploreront et fonde son propre culte en compagne de Ngasa (Cory, 1955). Plus loin encore de la zone de domination hinda, chez les Nyamwezi, le caractère extravagant et hors la loi de Wamara s'accentue. Il a épousé une princesse hinda et vit à la cour du roi de Karagwe. Ryangombe est l'un de ses favoris, de même que Mugasa et Lubinga. Wamara qui entre en conflit en matière religieuse avec le roi hinda se retire chez les siens, au Nkore où il se lie avec une bande de brigands. Il devient voleur de bétail. Les Hinda refusent d'accepter la doctrine religieuse de Wamara. Bientôt Ryangombe se sépare de Wamara, menacé par un présage : il s'en va à la chasse. Wamara et ses fidèles disparaissent dans un tremblement de terre et Ryangombe, qui a survécu au désastre, se mue en sauveur. Il fait annoncer qu'il guérirait tous ceux qui 
l'imploreraient, et serait «le libérateur de tous ceux qui sont attachés ». Mais il joue d'astuce. Il se présenta comme un Hinda et enjoint à ses fidèles de rendre un culte à l'ancêtre éponyme Ruhinda, le fondateur de la nouvelle dynastie. C'est sous cette couverture officielle que le nouveau culte se répand. Mais celui-ci est manifestement en rupture de ban par rapport à l'ordre officiel hinda puisque Ryangombe recommande à ses fidèles de voler, de commettre l'adultère, de tenir des propos impudiques, de mendier, d'être gloutons, de braver toute autorité. A sa mort, ses fidèles annoncent qu'il est parti préparer dans l'au-delà (dans la région des volcans situés dans le nord -ouest du Rwanda) un lieu de séjour dont l'accès serait interdit aux non-initiés (Bosch, 1930, pp. 202-207).

Ce récit prolonge directement la version sumbwa du mythe que je viens d'évoquer puisqu'il fut rédigé en langue sumbwa par un Tutsi du Nyamwezi. Les Tutsi sont des pasteurs dominants au Rwanda et le culte de Ryangombe leur est attribué en pays sumbwa. Selon une croyance largement répandue en Tanzanie, le culte des esprits swezi serait originaire de l'est, c'est-à-dire du Rwanda ou du Burundi (L. de Heusch, 1966, p. 284).

Mais curieusement, ni au Rwanda, ni au Burundi, Ryangombe, sauveur universel, n'est présenté comme un membre de la caste tutsi dominante. En fait, il est en Tanzanie (au Sumbwa comme au Nyamwezi) un rebelle hostile - au même titre que Wamara - au clan dynastique hinda. Il fait donc figure de troublion. Et c'est cette fonction de contestation mystique par rapport à l'ordre royal tutsi qu'il assume au Rwanda.

Lorsqu'il affronte le roi Ruganzu Ndori (qui est peut-être, rappelons-le, lui-même d'origine hinda), Ryangombe le vainc, non par la force des armes, mais par son pouvoir magique (L. de Heusch, 1982, pp. 207-210). J'ai suffisamment analysé ailleurs la démesure anarchique de Ryangombe et de la petite troupe d'ilotes qui l'accompagnent pour ne pas y revenir (L. de Heusch, 1982, chap. V). Bien que le culte soit représenté à la Cour, une règle rigide interdisait au souverain du Rwanda d'être initié aux mystères du « roi » Ryangombe. L'interprétation qu'en propose l'abbé Kagame me paraît décidément trop faible : les initiés doivent se prosterner devant le medium qui incarne Ryangombe lors des cérémonies; or une telle attitude signifierait que le roi se soumet à un autre, ce qui est évidemment inconcevable (Kagame, 1963, p. 62). Le roi Ruganzu Ndori n'a-t-il pas vu cependant, selon le mythe évoqué il y a un instant, les armes de ses guerriers se briser par une intervention magique décisive de Ryangombe ? Et, en instituant son culte au moment de sa mort, celui-ci n'abolit-il pas les différences de caste? Au début du XX ${ }^{\mathrm{e}}$ siècle, un membre de la famille royale ne déclarait-il pas à propos du culte de Ryangombe : « Nous laissons le travail (cultuel) aux Hutu (la caste paysanne). Les Tutsi ne violent pas leurs traditions. Ni le roi, ni les grands Tutsi ne peuvent devenir des Imandwa » (Loupias, 1908, p.6). Berger reconnaît elle-même que ce n'est qu'à une époque toute récente qu'un certain nombre de hauts personnages, y compris la reine-mère, se firent initier, le kubandwa étant impliqué dans des intrigues de cour (Berger, 1981, p. 84).

Cette distance structurale entre la royauté réelle et la royauté mystique de RyangombeKiranga est moins nette au Burundi. Il est bien vrai que le culte est parfaitement officialisé puisque, à la Cour, un dignitaire féminin, Muka-Kiranga, considéré comme l'épouse de Kiranga, joue un rôle dominant dans le rituel royal des prémices. Elle est considérée comme l'égale du souverain régnant (Berger, 1981, p. 84). Mais il n'en est pas moins tout aussi vrai que Muka-Kiranga personnifie la paysannerie hutu, lorsque son médium présidant aux travaux des champs souligne l'opposition à l'ordre pastoral dominant en injuriant les vaches et en les chassant des champs (Zuure, 1929, p. 48 et 
Bourgeois, 1956, p. 98). Les rois ne consultent pas les esprits du kubandwa et ne rendent pas de culte à Ryangombe-Kiranga (Coupez, 1957, p. 627)). Cette information précieuse, recueillie par Coupez de la bouche d'un informateur rundi, met en doute l'affirmation d'I. Berger selon laquelle « les initiés peuvent accéder à la royauté » (Berger, 1981, p. 77).

Sur ce terrain, Iris Berger me cherche une nouvelle querelle. Elle refuse mon interprétation selon laquelle le kubandwa serait au Rwanda une contestation symbolique de l'ordre royal tutsi. J'avais déjà essuyé précédemment les foudres de Claudine Vidal (Vidal, 1967) sous prétexte que l'initiation présente des parallèles frappants avec l'investiture royale. Mais comment s'en étonner alors que le kubandwa offre à toutes les catégories sociales, aux hommes comme aux femmes, la possibilité de s'identifier à un roi mystique, plus efficace sur le plan magique que le lointain roi terrestre? Le kubandwa évidemment ne prêche pas la révolution, mais un contre-ordre royal imaginaire. Reprenant la critique de Claudine Vidal à son compte, Iris Berger s'étonne cependant que j'ai pu affirmer que «la tension vers l'autonomie, l'indépendance, la survirilité s'affirme plutôt au niveau du langage qu'au niveau des actes» (L. de Heusch, 1966, p. 195). Insensible aux processus symboliques - qui n'entrent pas apparemment dans la conception qu'elle se fait de l'histoire - Berger estime que décidément je détruis (undermine) ici ma propre argumentation ${ }^{4} . .$. En outre, en interprétant le kubandwa comme je le fais, j'ignorerais que «la thématique du renversement symbolique et du désordre institutionnalité peut servir à renforcer plutôt qu'à contester les catégories classificatoires » (Berger, 1981, p. 82). Et voilà que l'argument fonctionnaliste vient tout arranger !

\section{Wamara et Ryangombe selon I. Berger}

Rejetant toute interprétation structurale des mythes, Berger est résolument partisane d'une histoire des religions éclatée, indifférente au sens. L'histoire du culte des Cwezi qu'elle nous propose s'effectue en effet au prix d'une perte sémantique considérable.

La geste cwezi ne serait qu'un ensemble hétéroclite de traditions claniques régionales. Le processus historique qu'elle tente de reconstituer, puisqu'elle est historienne - et seulement historienne - est celui de la «nationalisation d'esprits qui n'avaient auparavant qu'une importance locale et l'utilisation de leurs pouvoirs mystiques pour renforcer les Etats » (Berger, 1981, p. XIII).

41 Selon Berger, les conquérants bito et hinda se trouvèrent partout confrontés à l'opposition des autorités politiques autochtones qui s'exprimaient à travers leur divinité propre, un esprit cwezi. Celui-ci n'a aucune autre fonction historique ou mythique que celle de représenter un ancien clan dominant. Thèse tout arbitraire, radicalement fonctionnaliste...

42 C'est ainsi que le culte de Ndahura qui avait son siège principal à Mubende Hill « appartenait » au clan Sazima, qui fournissait la prêtresse de son temple. Elle jouait un rôle important dans les cérémonies de couronnement des rois nyoro (Berger, 1981, p. 33). Quant à Wamara, son lieu de culte principal se trouvait plus au sud, à Masaka Hill, dans le Bwera. Dans cette région qui recevait des pélerins venus de l'ensemble de l'Ouganda occidental, le clan des Moli a longtemps conservé une indépendance virtuelle. Voilà donc le clan moli déclaré propriétaire du culte de Wamara, sans aucune considération pour les liens structuraux que cette figure mythique entretient avec la précédente. Isaza, quant à 
lui, « représenterait » le clan sita, qui est toujours le plus important numériquement au Nyoro et au Toro (idem, p. 33).

Arrivons-en à Ryangombe. Il s'agirait, selon Berger, d'une très ancienne divinité locale associée au clan kirimbiri dans le sud-ouest de l'Ouganda, non loin de la frontière du Rwanda. Ces liens sont indéniables et l'on sera reconnaissant à l'auteur d'avoir sorti de l'ombre la figure du « roi des Imandwa ». Souverain mystique du Rwanda, Ryangombe fait aussi figure, en Ouganda, de roi d'un petit Etat, le Gitara, situé entre le Mpororo et le Ndorwa ; il était membre du clan dominant kirimbiri, qui est toujours représenté dans le district d'Igara au nord du Mpororo (Berger, 1981, p. 59, d'après une étude inédite de $\mathrm{F}$. Géraud). Mais, se fondant sur la même source, Berger évoque aussitôt après le témoignage d'un informateur du Nkore qui déclare : « le Cwezi Wamara a donné le tambour Murorwa à Babinga, le père de Ryangombe, pour qu'il exerce le gouvernement en son nom sur le Mpororo Rukiga ». On ne peut douter dès lors que le personnage de Ryangombe fasse partie de la geste des Cwezi. C'est d'ailleurs ce que dit expressément l'abbé Kagame (non cité par Berger) en confirmant le lieu d'origine du héros : Ryangombe et ses compagnons sont des Cwezi (Ibicwezi), qui se seraient livrés à un suicide collectif pour ne pas survivre à la mort de leur chef (Kagame, 1963, p. 62) ; ils viennent « du Gitara », région du royaume actuel du Toro, en Ouganda. «Ils étaient, précise-t-il encore, du clan des Cwezi (Abacwezi) lequel régna sur une vaste aire de ce dernier pays » (Kagame, 1963, p. 61). On notera qu'il existe un doute sur la localisation de ce «royaume» de Gitara, Kagame le situant, non près du Mpororo, c'est-à-dire non loin de la frontière rwandaise, mais plus au nord, au Toro. En tout état de cause, le nom Gitara n'apparait pas dans l'histoire du Nkore entreprise par Karugire qui inclut cependant celle du petit royaume de Mpororo, longtemps indépendant (Karugire, 1971).

Berger se garde bien de tirer parti de cette autre information, recueillie par Delmas, car ce serait donner raison à ma thèse : "Wamara est Ryangombe et les Cwezi (Abacwezi) sont les Imandwa des Nkore (Banyankole). Ils sont les mêmes que les nôtres, seuls quelques noms ont changé » (Berger, 1981, p. 58).

A vrai dire, Berger ne peut nier l'existence de liens historiques entre la geste des Cwezi et le mythe de Ryangombe ; mais elle est bien en peine de les expliquer. Elle se rallie aussi, sans le reconnaître explicitement, à ma thèse selon laquelle le culte des Cwezi-Imandwa s'est introduit au Rwanda à partir d'une région située quelque part au sud du pays haya. Je lui suis reconnaissant d'apporter ici à mon argumentation un argument nouveau de poids : le clan kirimbiri auquel, on l'a vu, appartient Ryangombe, se retrouve au Buha, sur les confins méridionaux du Burundi (Berger, 1981, p.59, d'après des archives territoriales). Cette information est confirmée par J.H. Scherer; dans trois des six petits royaumes ha, les rois (tutsi) appartenaient au clan kirimbiri (Richards, 1960, p. 212). Berger en conclut à juste titre que le clan kirimbiri, originaire du sud-ouest de l'Ouganda, a pu jouer un rôle dominant dans la diffusion du culte de Ryangombe dans l'aire méridionales de la civilisation interlacustre. Elle ajoute qu'un second clan pastoral, celui des Yango, jadis dominant au Karagwe, aurait contribué à ce processus. Mais cette hypothèse, je l'avais formulée dès 1966 (L. de Heusch, 1966, p. 343). Je rappelle brièvement mon argumentation fondée sur la comparaison des sources haya et rwandaises. Chassé du Karagwe par les conquérants hinda (encore eux !), un groupe d'aristocrates yango (sans doute hima) pénétra dans l'est du Rwanda où il donna une dynastie au petit royaume de Gisaka, qui conserva longtemps son indépendance. D'abord réfugiés au Bugufi (une région située au carrefour du pays haya, du Rwanda et du Burundi), les Yango ont très 
probablement contribué à l'introduction du culte de Ryangombe dans ces deux derniers royaumes. Je m'appuyais notamment sur le fait que les Yango observent la même interdiction totémique que Ryangombe-Kiranga au Burundi : le singe nkende. Par ailleurs, les traditions orales du Rwanda et du Burundi relatives au lieu d'origine du culte pointent vers la même direction: le sud du premier royaume et du nord du second (L. de Heusch, 1966, p. 342). Mais pas plus au Gisaka qu'au Rwanda, la tradition orale ne rattache la dynastie yango à Ryangombe. Il faut donc en conclure que la religion populaire centrée sur ce dieu qui meurt pour créer une religion de salut universelle était déjà constituée sous la forme de mystères initiatiques avant son introduction au Gisaka d'abord, au Rwanda méridional et central ensuite.

Seule la comparaison des rituels initiatiques dans l'ensemble de la civilisation interlacustre permet de résoudre cette énigme. Je résume mes conclusions, que Berger récuse sans raison valable. Il faut distinguer de ce point de vue trois sous-groupes dans l'aire Ryangombe : le sous-groupe sumbwa-nyamwezi (incluant sans doute une partie du Buha), le sous-groupe rundi (incluant probablement le Buha méridional), et le sousgroupe Rwanda ${ }^{5}$. J'insistais sur le fait que cette division correspondait grosso modo aux variations du mythe. Dans le premier cas, Wamara et Ryangombe coexistent, mais se séparent, Ryangombe assurant seule le rôle de fondateur d'un culte révolutionnaire ; dans le second, Ryangombe est plus généralement connu sous le nom de Kiranga et le culte, bien que d'inspiration démocratique, est étroitement associé aux rites royaux. Dans le troisième cas enfin - au Rwanda - Ryangombe, qui est seul en scène, comme au Burundi, concurrence nettement le pouvoir royal sur le plan mystique et dénie les différences de castes. Berger conteste le bien fondé de cette division sous prétexte qu'elle est fondée sur la continuité (illusoire à ses yeux) d'une culture cwezi au nord et au sud de la zone interlacustre (Berger, 1981, p. 66). Je dois donc m'expliquer. S'il y a continuité, c'est bien dans l'ordre mythique, chaque région offrant une permutation des données fournies par la geste des Cwezi. Cette argumentation resterait valable quand bien même Berger - et d'autres - auraient raison de contester la réalité historique d'une ancienne dynastie cwezi.

Mais il y a plus. Du point de vue strictement historique -c'est-à-dire sur le plan même où se confine mon contradicteur - la frontière méridionale de l'expansion hinda est un lieu critique. Elle me reconnaît même le mérite d'avoir découvert que la légende de Ryangombe comportait une forte composante anti-hinda (Berger, 1981, p. 66). Mais n'estce pas précisément le clan yango, déchu par les Hinda au Karagwe, qui aurait contribué à l'introduction des mystères de Ryangombe au Rwanda?

Or il se fait que le Bugufi, où le clan yango se serait réfugié après son exil du Karagwe, occupe une position géographique charnière entre les trois sous-groupes rituels que j'ai cru pouvoir isoler. Il y a donc lieu de croire que c'est sur les confins de l'expansion hinda (là où les rois cessent d'intégrer la tradition cwezi dans leur propre passé historique) que s'est effectué le passage de Wamara, le roi cwezi, à Ryangombe le rebelle, roi des Imandwa. Les divergences entre les rituels des trois sous-groupes du culte de Ryangombe suggèrent aussi que des évolutions historiques divergentes se sont produites à partir d'un même foyer, que l'on peut situer sur la frange sud du pays haya : dans une zone englobant le Bugufi, le Bushubi et le Buzinza. Celle-ci est située en effet entre les royaumes haya (placés sous le signe de Wamara) et le Sumbwa-Nyamwezi, où Ryangombe domine. Au Rwanda et, dans une moindre mesure, au Burundi, en pays tutsi, les mystères de Ryangombe perdent leur coloration anti-hinda, sans cesser pour autant de se présenter 
comme une religion de contestation, rivalisant avec le pouvoir magique des Rois. L'histoire décidément ne peut se passer de prendre en considération les données de l'ethnographie et de les interpréter.

Berger voit fort bien que dans l'aire septentrionale, le culte des Cwezi se déroule dans des temples dédiés à une divinité particulière où les cérémonies sont conduites par des prêtres et des médiums qui constituent un petit groupe de spécialistes, alors que dans l'aire méridionale apparaissent des cultes de possession largement ouverts à tous (Berger, 1981, p. 68). Mais elle n'aperçoit pas que partout l'accession au statut de médium possédé implique une initiation dérobée au regard, où apparaissent un certain nombre d'éléments récurrents. Le fait qu'au Nyamwezi, ces initiés soient membres d'une société secrète participant à la compétition pour le pouvoir, alors qu'au Rwanda, ils entrent dans une vaste confrérie religieuse jouissant d'un statut purement religieux, appelle sans doute une explication sociologique. Mais ces variations historiques ne doivent pas nous aveugler. Il s'agit bien, quoi qu'en pense Berger, d'un seul et même ensemble structural fondé sur la communication avec les dieux par le truchement de la possession. Il n'y a aucune raison de dissocier de cet aspect rituel - décrit par Berger comme un substrat commun - les deux grands systèmes de croyances, centrés respectivement sur Wamara et Ryangombe.

\section{Ryangombe et ses comparses}

De nouvelles questions se posent depuis que fut écrit mon livre. On peut se demander en particulier si le mythe de Ryangombe n'entretient pas au Burundi des rapports très lointains avec la geste relative à un peuple autochtone disparu : les Renge. Ici Ryangombe porte plus fréquemment le nom de Kiranga, son père est appelé Babingo ba Nyundo et son grand-père Birenge ba Nyundo (Zuure, 1929, p. 39). Le terme Birenge renvoie immédiatement aux Renge et nyundo désigne le marteau de forge. Au Rwanda aussi, Babinga le père de Ryangombe est un descendant de Nyundo (Sandrart, 1939, deuxième partie, p. 57). Berger fait remarquer à ce propos que les traditions du Rwanda associent la dynastie renge, qui régnait jadis sur la moitié occidentale du pays, à la forge (Berger, 1981, p. 37). Il se pourrait donc que Kiranga soit au Burundi le produit syncrétique d'une ancienne divinité chthonienne locale avec la figure (évidemment cwezi) de Ryangombe. Un premier informateur de Vansina soutient effectivement que le culte du « roi » Kiranga a absorbé d'anciennes liturgies transmises par les Renge (Vansina, 1972, p. 105). Mais un autre est parfaitement conscient de l'origine septentrionale du culte : il affirme que les Cwezi (ibicwezi), les suivants de Kiranga, quittèrent le Nkore, son pays d'origine (Vansina, 1972, p. 104). Il est remarquable que l'initiation au culte ait conservé au Burundi un trait capital de l'intronisation du souverain du Nkore : le baptême dans la rivière, trait qui fait défaut dans le rituel de Ryangombe au Rwanda (Roscoe, 1923, p. 57; Zuure, 1929, p. 63).

On ne peut douter que Kiranga ne soit un doublet de Ryangombe; grand chasseur, il meurt comme lui tué par un buffle ou une antilope (Vansina, 1972, p. 104). Mais la présence dans le culte de Kiranga de nombreux esprits inconnus au Rwanda confirme l'impression que le kubandwa est un culte syncrétique, absorbant d'anciennes divinités. C'est sans doute ce qui explique sa reconnaissance comme religion nationale. Le médium qui incarne à la cour Muka-Kiranga, la femme du dieu associée à l'agriculture, est considéré comme l'égal du souverain règnant (Gorju, 1938, p. 45) et généralement, selon l'observation de Berger, les mythes décrivent des relations de coopération entre Kiranga 
et le souverain (Berger, 1981, p. 80) bien que, pas plus qu'au Rwanda, celui-ci ne rende un culte à celui-là (Coupez, 1957).

Le phénomène du syncrétisme joue aussi au Rwanda. J'ai montré dès 1966 que le thème de la dernière chasse tragique de Ryangombe s'inspirait directement de la chronique légendaire royale décrivant le suicide du roi Bwimba Ruganzu, destiné à sauver le pays (L. de Heusch, 1966, pp. 253-256 ; 1982).

Une autre question nouvelle surgit: pourquoi Ryangombe se trouve-t-il fréquemment associé, dans l'aire culturelle méridionale, avec Mugasha? Cet esprit ne joue aucun rôle marquant dans la geste septentrionale des Cwezi, où il est présenté comme l'un des demifrères de Ndahura et oncle paternel de Wamara (Nyakature, 1973, figure II ; Schmidt, 1978, p. 63). Il s'agit apparemment d'une très ancienne divinité locale des eaux associée au lac Victoria, plus ou moins brouillée avec Ndahura. En effet, le mythe nyoro raconte que le roi des Cwezi l'aurait exilé dans l'une des îles du lac (Nyakatura, 1973, p. 31). Selon la version nkore, Mugasha est le seul Cwezi à ne pas disparaitre dans le lac Kayikambara : il s'installe dans les îles Sese (lac Victoria) où il vit toujours (Oberg, 1970, p. 124).

$\mathrm{Au}$ Ziba, Mugasha entre en conflit avec le roi Wamara dont il convoite la fille. Mais celle-ci est dégoûtée par ce pêcheur malpropre au statut inférieur. Mugasha argue qu'il était le maitre de toutes les créatures aquatiques. Il envoye un petit poisson auprès de Wamara pour obtenir la main de la belle. Mais cet ambassadeur est malmené. Alors Mugasha charge Nkuba la Foudre de combattre Wamara. Nkuba lance dans le palais une énorme pierre sur laquelle Wamara se réfugie tandis qu'une pluie diluvienne s'abat tout autour de lui. Il pleut pendant deux jours. Le palais est transformé en lac où jouent l'hippopotame et le crocodile. Effrayé, le peuple charge ces deux animaux de conduire la fille de Wamara auprès de leur maitre. Celui-ci confectionne pour elle un vêtement d'écailles de poisson. Alors Nkuba enlève le rocher et l'eau s'assèche. Kagoro, qui est absent, revient à la cour et combat Mugasha. Mais la flèche qu'il lui décoche dans le gras de la cuisse ne fait pas plus d'effet qu'un moustique. Mugasha l'assomme d'un coup de rame. Kagoro ramène cependant la fille de Wamara auprès de son père. Le lendemain matin, Mugasha vient à la cour implorer le pardon du roi. Il quitte son domaine aquatique avec tous ses biens. Il se réconcilie avec Wamara ou, selon d'autres versions, devient son serviteur. On construit pour lui une maison en dehors de l'enclos royal (Schmidt, 1978, pp. 70-72).

Fait remarquable, ce Cwezi quelque peu marginal a une relation privilégiée avec la dynastie du Ziba, qui est d'origine bito. Alors que généralement en pays haya, les dynasties hinda ont une relation positive avec Wamara, considéré comme leur ancêtre, le roi du Ziba est l'allié des médiums de Mugasha contre Wamara et les Cwezi (Schmidt, 1978, p. 66). La position excentrique du maître des eaux se trouve donc exploitée ici à des fins politiques. On comprend mieux qu'un rapprochement structural ait pu s'effectuer, ailleurs, entre ce Mugasha, puissant magicien mais de statut social inférieur, avec Ryangombe, le révolté, en rupture de ban avec Wamara. C'est ainsi que s'explique la curieuse association des deux esprits dans plusieurs versions du mythe. Au Nyamwezi, Ryangombe quitte Wamara en même temps que Mugasha: le premier s'en va chasser tandis que le second s'en va pêcher en compagnie d'un certain Lubinga. Ensemble Mugasha et Lubinga dérobent un grand nombre de vaches à Wamara qu'ils emmènent dans une île du lac Victoria. Ryangombe seul fonde un nouveau culte qui fait figure de religion de salut (Bosch, 1930, pp. 207-217). Au Sumbwa, loin de se séparer de Ryangombe, Mugasha est avec celui-ci le co-fondateur de ces mystères (Cory, 1955). Enfin au Rwanda, il se trouve être le gendre de Ryangombe. La légende qui le concerne n'est qu'une 
réplique du récit ziba présenté ci-dessus. C'est ce que nous apprend une note de terrain de Vansina, que je résume ici (Berger, 1981, p. 145).

Mugasa était un serviteur de Ryangombe avec qui il a de nombreux sujets de friction. Il enferma Kagoro dans un rocher et demanda en mariage Nyabibungo, la fille de Ryangombe. Mugasa l'emmena dans une île du lac Kivu et libéra Kagoro. Kagoro suggéra alors à Ryangombe de proposer au couple de venir vivre après des Imandwza en promettant à Mugasa qu'il ne serait plus jamais maltraité.

Cette version ne laisse pas de doute que Ryangombe est au Rwanda une permutation du Wamara de la légende ziba. Mugasa et Kagoro sont ici les rares compagnons de Ryangombe à faire l'objet d'un récit. Pierre Smith en analyse fort bien les traits distinctifs. Mugasa, «connu dans les royaumes ougandais », est « un personnage vulgaire et grossier, puant et méchant qui dégoûte tout le monde et exige des droits de passage pour la traversée des cours d'eau », alors que Kagoro, son adversaire, « représente l'idéal aristocratique, pastoral et guerrier ». Cette opposition possède une dimension cosmologique car, face à Mugasa esprit des eaux terrestres, Kagoro est « associé au feu du ciel » (Smith, 1975, pp. 74-75) ${ }^{6}$. Ryangombe complète enfin ce couple d'oppositions en introduisant un troisième terme structural : «Alors que Ryangombe 'aime trop les vagins' et prend n'importe quelle femme de rencontre, Kagoro est un raffiné à qui la plupart des filles soulèvent le coeur et qui ne pourra se marier, très tardivement, qu'avec la plus parfaite... » (Smith, 1975, p. 74). J'ai longuement décrit précédemment le caractère anarchiste de Ryangombe, roi-chasseur, opposé au modèle du roi-guerrier (L. de Heusch, 1982, pp. 196-213).

Mais Mugasa et Kagoro sont intégrés dans la famille de Ryangombe : si le premier est son gendre, le second est considéré comme son demi-frère ou l'un de ses fils (Arnoux, 1912, p. 282; Pagès, 1933, p. 364). Cependant, les traditions orales qui les concernent se séparent : le cycle de Kagoro, entouré de Preux, appartient à la littérature profane. Dans un récit rapporté par Smith, Kagoro affronte les Nyoro qui ont envahi le pays à l'époque des rois Mukobanya et Mutabazi : il combat un roi bito (Smith, 1975, pp. 263-283). La geste mythique devient ici chanson de geste.

thologie cwezi associe donc de manière privilégiée Mugasha (ou Mugasa) et Kagoro, deux divinités cosmologiques qui appartiennent sans doute à une très ancienne strate mythologique ${ }^{7}$. Mais dans la généalogie officielle des Cwezi du Nyoro, Mugasa figure comme oncle paternel de Wamara alors que Kagoro est son cousin parallèle patrilatéral (Nyakatura, 1973, figure II). Au Nkore, Mugasha figurait avec Wamara, Kagoro et une certaine Nyakiriro ${ }^{8}$ parmi les quatre Imandwa principaux à qui l'on rendait un culte en faveur du roi règnant sur un autel construit dans le palais de la reine-mère (Oberg, 1970, p. 161). Ils font partie des grands Cwezi, dont Ryangombe et quelques autres sont les serviteurs (Karugire, 1971, p. 84). Il est remarquable de constater que ces esprits de seconde catégorie sont loin de jouir du même prestige que les premiers. Karugire va même jusqu'à affirmer que l'on craint leur pouvoir de nuire. Les offrandes faites en leur honneur n'ont d'autre fonction que d'apaiser leur courroux pour mettre fin à une infortune qui leur est attribuée; jamais Ryangombe n'est invoqué en dehors de ces cas particuliers.

60 C'est donc bien à une transformation radicale de la figure de Ryangombe que nous assistons là où il se substitue au bienveillant Wamara, usurpant son titre royal. Ce processus s'accompagne partout de processus syncrétiques particuliers et l'idée de présenter le kubandwa comme une religion parfaitement homogène est évidemment tout 
à fait étrangère à mon propos. La mythologie cwezi - comme toute mythologie - est assurément le produit d'un bricolage historique, mais celui-ci opère selon une ligne de clivage structurale. Un dernier exemple. Dans la geste haya rapportée par Césard, Mugasha accompagne Wamara au cours de sa partie de chasse nocturne, qui le conduit dans le monde inférieur où il reçoit du bétail. Un troisième larron, Irungu, se joint à eux. Dans la quête de la richesse bovine qui est l'objet de cette partie du mythe, les personnages se complètent symboliquement. Si Mugasha est l'esprit des eaux terrestres (voleur de bétail selon la version nyanwezi), Irungu est l'esprit de la brousse, de la terre non cultivée. Ce sont donc bien les représentants du monde sauvage, non domestiqué, qui entourent Wamara dans sa folle équipée cynégétique qui le mettra en possession du signe majeur de la civilisation : le bétail. On comprend que Kagoro le raffiné ne se soit pas joint à eux. Il interviendra plus tard. Dans la geste nyoro et nkore, il a pour mission de récupérer le bétail volé aux Cwezi (Berger, 1981, p.143). Et dans la geste nyamwezi récoltée par Bosch, il se précipite à la poursuite de Mugasha qui vient de dérober le bétail de Wamara.

61 Wamara-Ryangombe, Mugasha et Kagoro forment donc dans l'aire méridionale de la civilisation interlacustre une espèce de trio structural, étranger à la geste cwezi au Nyoro. Cette élaboration est assurément le produit d'une histoire religieuse particulière, mais l'on voit mal quels conflits claniques pourraient en rendre compte.

62 C'est bien là la faiblesse de la thèse de Iris Berger. Son ambition est de nous persuader que la religion est un facteur de résistance dans cette partie de l'Afrique (c'est le titre même de son livre). Mais qu'en est-il en fin de compte? Examinons la conclusion de son ouvrage. De nouveaux conquérants se trouvent partout, affirme-t-elle, confrontés à l'opposition des autorités politiques établies qui s'expriment au nom de leur divinité propre. Dans certains cas, ces esprits étaient d'anciens symboles religieux autochtones; dans d'autres, des divinités étrangères présentant une forte réputation anti-dynastique (c'est tout particulièrement le cas de Ryangombe) (Berger, 1981, p. 89). Dans les deux cas, poursuit tranquillement l'auteur qui nage en pleine conjecture, ces oppositions à coloration religieuse « offraient aux dissidents le moyen de protéger leur propre position ou de mobiliser une résistance anti-gouvernementale ».

63 Voilà comment l'on peut faire, en s'appuyant sur une sociologie politique sommaire, l'économie du sens. Si l'histoire n'a pas, à proprement parler, de sens, elle infléchit cependant sans cesse les structures signifiantes, dont la religion fait partie, et elle doit donc être prise au sérieux. C'est ce que j'ai tenté de faire, là où l'entreprise ne me paraissait pas tout à fait désespérée. Mais l'histoire, telle que la pratiquent Berger et bien d'autres menace l'anthropologie dans ses fondements mêmes.

\section{BIBLIOGRAPHIE}

ADLER, Alfred, 1982 : La mort est le masque du roi. La royauté sacrée des Moundang du Tchad, Payot, Paris. 
BEATTIE, John, 1957 : «Initiation into the Cwezi Spirit Possession Cult in Bunyoro », African Studies, vol. 16, 3, pp. 150-161.

BERGER, Iris, 1981 : Religion and Resistance. East African Kingdoms in the Precolonial Period, Annales du Musée Royal de l'Afrique centrale, Tervuren.

BOSCH, Fridolin, 1930 : Les Banyamwezi : peuple de l'Afrique orientale, Munster.

CESARD, Edmond, 1927 : « Comment les Bahaya interprètent leurs origines », Anthropos, XXII, 3-4, pp. 440-465.

CESARD, Edmond, 1937 : « Le Muhaya », Anthropos, XXXII, 1-2, pp. 15-60.

CORY, Hans, 1955 : « The Buswezi », American Anthropologist, LVII, 5, pp. 923-952.

CORY, Hans et HARTNOLL, M.M., 1945 : Customary Law of the Haya Tribe, Londres.

COUPEZ, André, 1957 : « Texte ruundi », Zaïre, XI, 6, pp. 623-636.

d'HERTEFELT, Marcel, 1981 : Les clans du Rwanda ancien. Eléments d'ethnosociologie et d'ethnohistoire, Annales du Musée Royal de l'Afrique Centrale, Tervuren.

FORD, J. et HALL, R. de Z, 1947 : « The History of Karagwe (Bukoba District) », Tanganyika Notes and Records, XXIV, pp. 3-27.

GORJU, Julien et al., 1938 : Face au royaume hamite du Rwanda : le royaume frère de l'Urundi, Bruxelles.

HEUSCH, Luc de, 1966 : Le Rwanda et la civilisation interlacustre. Etudes d'anthropologie historique et structurale, Université Libre de Bruxelles.

HEUSCH, Luc de, 1972 : Le roi ivre ou l'origine de l'Etat, Gallimard, Paris.

HEUSCH, Luc de, 1982 : Rots nés d'un coeur de vache, Gallimard, Paris.

KAGAME, Alexis, 1963 : Les milices du Rwanda précolonial, Académie royale des Sciences d'OutreMer, Bruxelles.

KARUGIRE, Samwiri Rubaraza, 1971 : A History of the Kingdom of Nkore in Western Uganda to 1896, Clarendon Press, Oxford.

NYAKATURA, J.W., 1973 : Anatomy of an African Kingdom. A History of Bunyoro-Kitara, translated by Teopista Muganwa, edited by Godfrey N. Uzoigwe, Nok Publishers, New York.

OBERG, K., 1970 : « The Kingdom of Ankole in Uganda », in African Political Systems, ed. M. Fortes et E.E. Evans-Pritchard, Oxford University Press, Londres-Oxford-New York, pp. 121-162 (éditions originale 1940).

OLIVER, Roland, 1953 : « A Question about the Bacwezi », Uganda Journal, XVII, 2, pp. 135-137.

OLIVER, Roland, 1955 : «The Traditional Histories of Buganda, Bunyoro and Nkole », Journal of the Royal Anthropological Institute, LXXXV, 1-2, pp. 111-117.

OLIVER, Roland, 1959 : « Ancient Capital Sites of Ankole », Uganda Journal, XXIII, 1, pp. 51-63.

PAGES, Rév. Père, 1933 : Un royaume hamite au centre de l'Afrique, Institut Royal Colonial Belge, Bruxelles.

RICHARDS, Audrey I. (ed.), 1960 : East African Chiefs. A Study of Political Development in some Uganda and Tanganyika Tribes, Faber and Faber, Londres.

ROSCOE, John, 1923 : The Bakitara of Banyoro, Londres. 
SCHMIDT, Peter R., 1978 : Historical Archaeology. A Structural Approach in an African Culture, Greenwood Press, Westport (Connecticut), Londres.

SMITH, Pierre, 1975 : Le récit populaire au Rwanda, Armand Colin (Classiques africains), Paris. VANSINA, Jan, 1962 : L'évolution du royaume rwanda des origines à 1900, Académie des Sciences d'Outre-Mer, Bruxelles.

VANSINA, Jan, 1972 : La légende du passé. Traditions orales du Burundi, Musée Royal de l'Afrique Centrale, Archives d'Anthropologie, $n^{\circ} 16$, tervuren.

VAN NOTEN, Francis L., 1972 : Les tombes du roi Cyirima Rujigira et de la reine-mère Nyirayuhi Kanjogera, Annales du Musée Royal de l'Afrique centrale, Tervuren.

VIDAL, Claudine, 1967 : «Anthropologie et histoire. Le cas du Rwanda », Cahiers Internationaux de Sociologie, XLIII (juillet-décembre).

W.K., 1935 : « Abakama ba Nunoro-Kitara », Uganda Journal, III, 2, pp. 155-160.

WRIGLEY, C.C., 1973 : « The Story of Rukidi », Africa, XLIII, 3, pp. 219-235.

ZUURE, Bernard, 1929 : Croyances et pratiques religieuses des Barundi, L'Essorial, BruxellesElizabethville.

\section{NOTES}

1. Pierre Salmon à qui je dédie ces pages est une notable exception.

2. Prononcer tchwézi.

3. On comparera avec la figure du roi jumeau dans la royauté mundang du Tchad (Adler, 1982).

4. Plus subtilement, Marcel d'Hertefelt me suggère de parler plutôt de catharsis que de contestation (d'Hertefelt, 1981, p. 76).

5. Berger juge sans fondement la division que j'ai proposée entre le buha septentrional et le buha méridional (Berger, 1981, p. 66). Elle la reprend pourtant à son compte un peu plus loin en observant que l'on retrouve dans le nord du Buha, (et non dans le sud) une série de termes liturgiques qui mettent en relation cette religion avec la zone cwezi septentrionale (idem, p. 76).

6. On notera que dans le récit ziba, Nkuba la Foudre passe du côté de Mugasa dans son combat contre le guerrier Kagoro.

7. Le thème de l'opposition entre un génie (fruste) des eaux terrestres, opposé à un héros (hypercivilisé), maître de la Foudre, se retrouve dans la geste luba du Zaïre (L. de Heusch, 1972, chap. II).

8. On notera avec intérêt que cet esprit féminin se retrouve dans le kubandwa rwandais comme demi-soeur de Ryangombe (Sandrart, 1939, deuxième partie, p. 55).

\section{RÉSUMÉS}

The structural analysis of myth, far from being incompatible with historical interpretation, can in fact be a great aid to it. This article is in response to I. Berger's 1981 reproach of L. de Heusch Rwanda et la civilisation interlacustre (1966) for believing "that spirits, myths and rites form a 
unified system, reaching across time and space".

The cult of possession of the Kubandwa in the interlacustrian region of Central Africa is seen from both synchronic and diachronic points of view as a system of transformation. Despite new information brought to bear by I. Berger, the author here maintains the essential argument which he put forward in 1966: the myth and initiatory cult of Ryangombe in the southern part of the interlacustrian civilization come from the myth of Wamara and from the worship of Cwezi spirits in Nyoro and Nkore (Uganda).

History, as practised by I. Berger and others, is a threat to the very foundations of anthropology.

\section{AUTEUR}

\section{LUC DE HEUSCH}

Faculté des Sciences sociales, politiques et économiques - Université Libre de Bruxelles Belgique 